\title{
Evaluación de la materia orgánica con respecto a la altitud en sistemas agricolas del Tolima
}

\section{Evaluation of organic matter regarding altitude in agricultural systems from Tolima}

\author{
Blanca Myriam Salguero Londoño ${ }^{1}$ \\ Diego Mauricio Brínez Varón²
}

(2)

Recepción: 29/09/2020
(-)

Aprobación: 14/10/2020
(.)

Publicación: 18/12/2020

\section{Para citar este artículo:}

Salguero Londoño, B. M., \& Brínez Varón, D. M. (2020). Evaluación de la materia orgánica con respecto a la altitud en sistemas agrícolas del Tolima. Indagare, (8), 137-144. https://doi.org/10.35707/indagare/811

\section{(c) $\underset{\mathrm{BY}}{\mathrm{N}} \mathrm{\Theta}$}

\footnotetext{
${ }^{1}$ Grupo de investigación Naturatu, Universidad de Ibagué, Colombia. ORCID: 0000-0003-0508-8753. Correo electrónico: blanca.salguero@unibague.edu.co

${ }^{2}$ Grupo de investigación Naturatu, Universidad de Ibagué, Colombia. Correo electrónico: 520151003@estudiatesunibague. edu.co
} 


\title{
Resumen
}

El objetivo del trabajo fue evaluar la dinámica de la materia orgánica (MO) con respecto a la altitud en diez fincas cafeteras ubicadas al norte y sur del departamento del Tolima. Se evaluó la respiración basal (RB) y algunas propiedades químicas de los suelos estudiados, para determinar la existencia de un posible efecto de estas sobre la MO. Los valores que se registraron para el contenido de MO cuentan con un mínimo de $2 \%$ y un máximo de $5 \%$. Al analizar su comportamiento y ser sometidos a un análisis de varianza, se demuestra que para los resultados de la investigación no se encuentra una relación entre las variables MO y pisos altitudinales. Por otra parte, se considera que las variables de humedad, temperatura, capacidad de intercambio catiónico (CIC) y $\mathrm{pH}$ influyen y se relacionan con los resultados del contenido de la MO en el suelo.

\begin{abstract}
The objective of this work was to evaluate the dynamics of organic matter (OM) regarding altitude in ten coffee farms located in the north and south from the department of Tolima. Basal respiration (BR) and some chemical properties of the studied soils were evaluated to determine the existence of a possible effect of these on $\mathrm{OM}$. The values that were registered for the om content have a minimum of $2 \%$ and a maximum of $5 \%$. When analyzing their behavior and being subjected to an analysis of variance, it is shown that for the results of the research there is no relationship between variables OM and altitudinal floors. On the other hand, it is considered that the variables of humidity, temperature, cationic exchange capacity (CEC) and $\mathrm{pH}$ influence and are related to the results of the OM content in the soil.
\end{abstract}

Palabras claves: Respiración basal, altitud, humedad, indicador.
Key words: Basal respiration, altitude, humidity, indicator.

\section{Introducción}

El suelo es un ecosistema importante para la humanidad por los servicios ecosistémicos que presta, entre los cuales se encuentra la producción de biomasa. Sin embargo, el uso de sistemas intensivos de producción ha generado un impacto significativo para él, lo cual despierta un interés entre investigadores y tomadores de decisiones en cuanto a la gestión de su uso. Algunos países como Argentina, donde representa una parte importante de sus ingresos y el motor de la economía, han incursionado en diferentes prácticas para elevar su productividad como lo ha hecho durante los últimos años. En estos periodos 
se han implementado estrategias basadas en la evaluación de sus propiedades físicas y químicas, obteniendo resultados en el incremento de su producción en un $15 \%$. Lo anterior se debe a la incorporación de nuevas hectáreas de sus cultivos y un 85 \% por la adopción de tecnología: genética de semillas y mayor conocimiento, y control acerca de las propiedades físicas, químicas y biológicas (Esperbent, 2018).

La región alto-andina de Colombia, se caracteriza por presentar sistemas agrícolas de pequeños productores, que, por encontrarse en zonas altas del país, deben enfrentarse a numerosas adversidades debido a la variación del clima que influye en las propiedades físicas, químicas y biológicas del suelo y que afectan la productividad de sus cultivos (Acevedo-Osorio, Angarita-Leiton, León Durán \& Franco Quiroga, 2017). La MO se ha convertido en uno de sus principales componentes estudiados, en ella se analiza su cantidad, calidad y su interacción con el terreno, y se considera un indicador de calidad por su variedad de componentes como el fósforo $(\mathrm{P})$, el nitrógeno $(\mathrm{N})$ y potasio $(\mathrm{K})$, que aportan a la fertilidad, nutrición y calidad del suelo (Farrus-Miró, 2016). El objetivo de la presente investigación fue evaluar la dinámica de la cantidad de MO con respecto a la altitud y su relación con la RB en algunos suelos de sistemas agrícolas del departamento del Tolima.

\section{Materiales y métodos}

Se seleccionaron diez sistemas agrícolas ubicados en el departamento del Tolima con diferencias altitudinales y muestreados una vez en el mes de febrero de 2020. Los sistemas agrícolas seleccionados cuentan con una extensión aproximada de cinco a treinta hectáreas, clasificados como pequeños y medianos productores. Los productores que participaron en la investigación pertenecen al proyecto de Implementación de innovación de procesos a nivel de poscosecha para la incorporación de valor agregado en la diferenciación de cafés especiales del Tolima. Durante la investigación se tomaron un total de veinte datos para obtener los resultados de las variables ( $\mathrm{MO}, \mathrm{RB}, \% \mathrm{H}, \mathrm{pH}$ y CIC) en diferentes pisos altitudinales (1180 m s. n. m. -1800 m s. n. m.) en dos zonas del departamento del Tolima en un periodo de la época seca en febrero de 2020.

Dentro de cada sistema agrícola se seleccionaron dos lotes al azar. Luego, dentro de cada finca, se procedió a elegir diez sitios de muestreo que correspondían a la toma de las diez submuestras homogéneas de suelo que se mezclaron entre sí para dar origen a una muestra compuesta. La selección de la toma de submuestras se realizó por el método de grilla, el cual consiste en generar puntos aleatorios en un polígono. En este caso, el lote, previamente georreferenciado y cargado en el software Arcgis y Excel, permitió localizar los puntos en el Sistema de Posicionamiento Global (GPS). La toma de muestras de suelo 
INDAGA3E e-ISSN: 2357-5042 • Número 8 (2020) • Universidad de Ibagué • doi: https://doi.org/10.35707/indagare/811

se realizó a una profundidad aproximada de $20 \mathrm{~cm}$ con el uso de un barreno holandés y la ayuda de un palín, siguiendo la guía de toma de muestras de suelo propuesta por la Corporación Colombiana de Investigación Agropecuaria (Corpoica).

\subsection{Análisis de laboratorio}

Se utilizó el método Walkley y Black modificado para determinación de MO y el método de Isermeyer de frascos cerrados para la determinación de la RB. Para la determinación de MO del suelo, según el método de Walkley y Black modificado (combustión húmeda), se pesaron 0,25 g de muestra tamizada de suelo $(2 \mathrm{~mm})$, y se depositó en un erlenmeyer de 250 $\mathrm{mL}$. Posteriormente, se agregaron $5 \mathrm{~mL}$ de solución de dicromato de potasio $\left(\mathrm{K}_{2} \mathrm{Cr}_{2} \mathrm{O}_{7}\right)$ en medio ácido y $10 \mathrm{~mL}$ de ácido sulfúrico $\left(\mathrm{H}_{2} \mathrm{SO}_{4}\right)$ concentrado, agitando vigorosamente durante un minuto. Se dejó en reposo durante treinta minutos y al alcanzar la temperatura ambiente se adicionaron $5 \mathrm{~mL}$ de ácido fosfórico $\left(\mathrm{H}_{3} \mathrm{PO}_{4}\right)$ y se completó a volumen de $150 \mathrm{~mL}$ con agua destilada y se agitó de nuevo.

La solución se dejó decantar y enfriar durante dos horas y el sobrenadante se pasó a tubos de ensayo $(10 \mathrm{~mL})$. Se dejó en reposo durante veinticuatro horas y se fijó la absorbancia a $590 \mathrm{~nm}$, debido a que la intensidad del color es cuantificada en dicha longitud de onda. Después, se construyó la curva de calibración a partir de una solución patrón de sacarosa al 5 \%. Se prepararon las soluciones de trabajo: de la solución patrón se tomaron alícuotas de 5,10,15,20,25,50 mL, se depositaron en balones aforados de 100 $\mathrm{mL}$ y se llevaron a volumen. De cada una de las soluciones preparadas se tomaron $2 \mathrm{~mL}$ y se siguió el mismo procedimiento descrito para las muestras de suelo (García Galvis \& Ballesteros González, 2005). Por otra parte, se realizaron análisis físicoquímicos de las muestras que fueron llevados a cabo en el Laboratorio de Servicios de Extensión en Análisis Químicos (Laserex) de la Universidad del Tolima.

\section{Resultados}

Los datos MO, RB, humedad $(\% \mathrm{H})$ y CIC fueron analizados por medio del software InfoStat versión 2020, con el fin de comparar los resultados de las variables biológicas (MO y RB) con las demás variables. Además, se realizó un análisis de componentes principales (ACP) entre las variables MO y altitud para establecer su grado de asociación. Para la MO, se realizó una comparación mediante un gráfico de barras en el que se proyectaron los resultados del contenido con respecto a la altitud en la que se registró cada una de las muestras, con el fin de observar, espacialmente, el comportamiento de dicha variable (Figura 1). 
Figura 1. Cantidades de MO en los sistemas muestreados a diferentes pisos altitudinales en los municipios objeto de estudio, febrero 2020

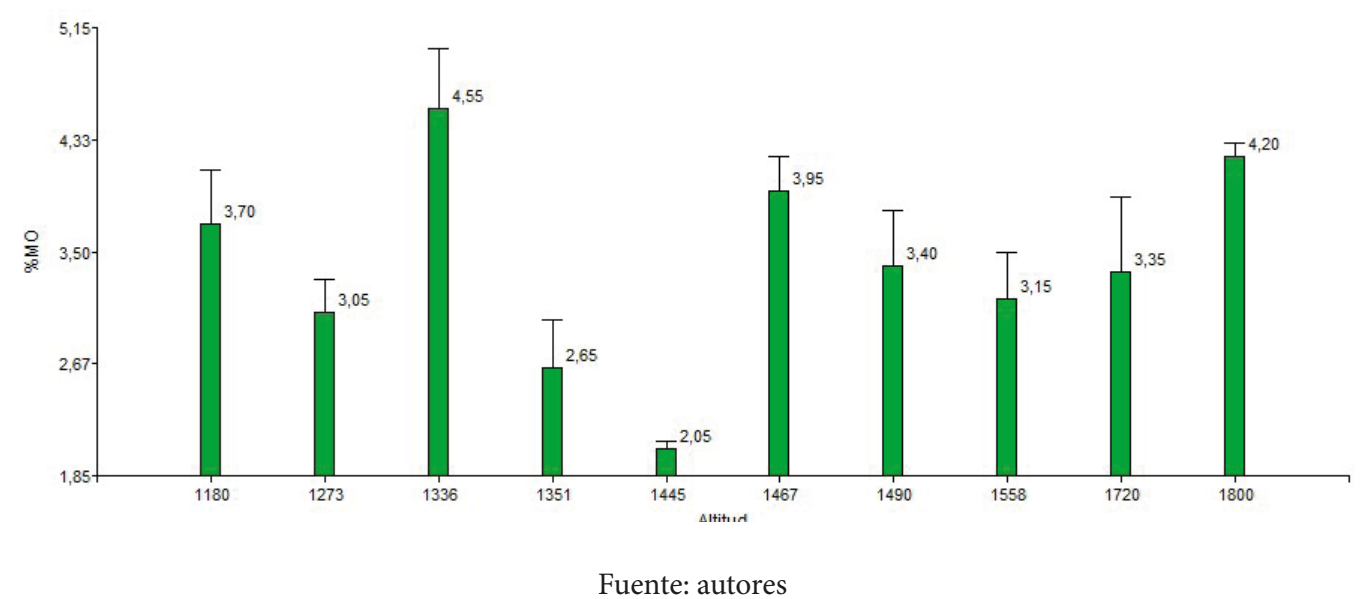

En la Figura 1 se observa la variable MO y su comportamiento con respecto a los diferentes gradientes altitudinales muestreados, en donde se analiza que no hay un comportamiento que demuestre una relación entre las variables, dado que no aumenta ni disminuye la variable MO con el movimiento de los diferentes gradientes altitudinales.

Para el desarrollo del proyecto, se tuvo en cuenta el factor altitud, puesto que se ha reportado, según Puerta Quintero et al. (2016), que el porcentaje de MO varía con esta condición, porque registra una relación positiva entre el contenido de materia orgánica en el suelo (MOS) y las variables de humedad y altitud. Lo anterior se presenta debido a que mientras hay un aumento de los pisos altitudinales surge una reducción en la temperatura que conlleva aumentar el porcentaje de humedad $(\% \mathrm{H})$ e influye en la presencia de la MO, porque al reducir la temperatura la digestión de la MO disminuye y la humedad conserva esta variable que mejora la fertilidad y la producción de los cultivos.

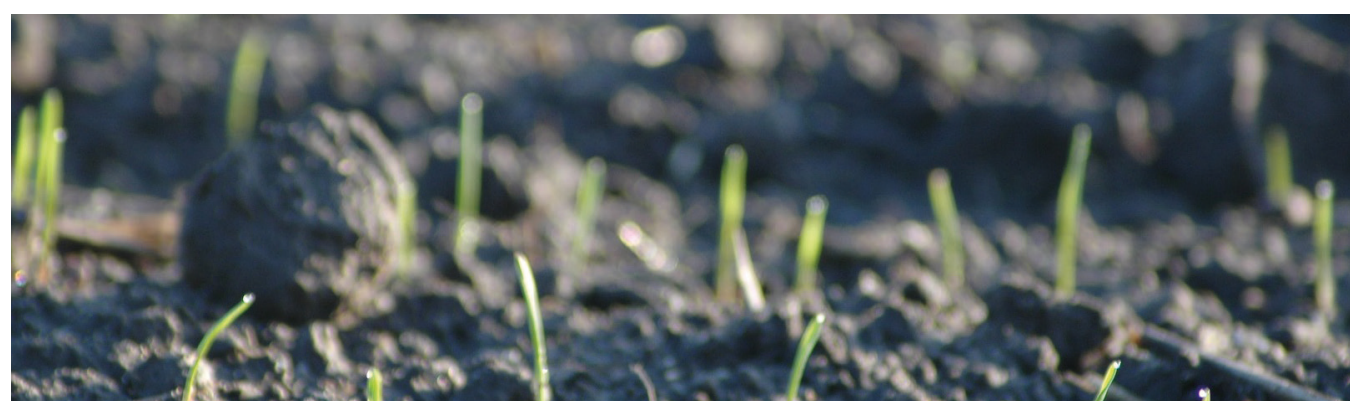


Figura 2. Resultados de la prueba de RB para las fincas ubicadas en la zona sur para febrero 2020

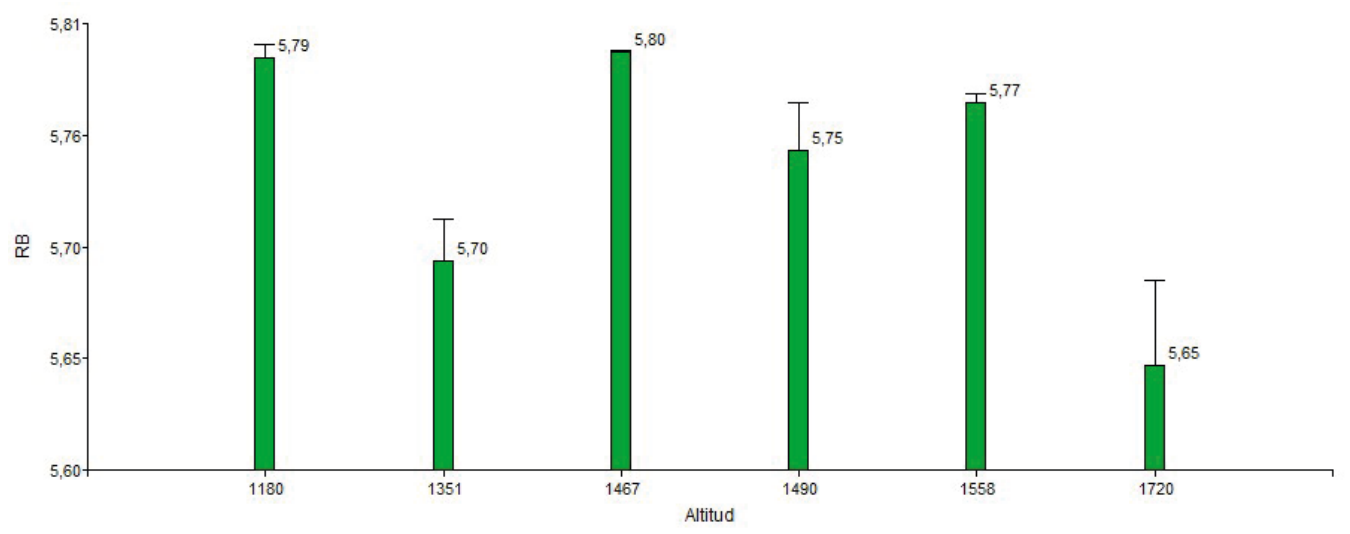

Fuente: autores

En la Figura 2 se observa el comportamiento de la variable RB con respecto a la altitud, no hay una relación entre las variables debido a que no hay una tendencia al aumento o disminución RB. Las medidas resumen que la variable RB en la zona sur presentó un valor promedio de 5,74 $\mu \mathrm{g}$ C-CO2 /gss, un valor mínimo de $5.61 \mu \mathrm{g}$ C-CO2 /gsc y un valor máximo de $5.80 \mu \mathrm{g}$ C-CO2 /gsc y una desviación estándar de 0.06. Para la zona norte (Figura 3), los resultados nos permiten observar que no hay una relación entre las variables debido a que en la zona sur no hay una tendencia al aumento ni a la disminución de la variable RB con respecto a los diferentes gradientes altitudinales.

Figura 3. Resultados de la prueba de RB para las fincas ubicadas en la zona norte para febrero 2020

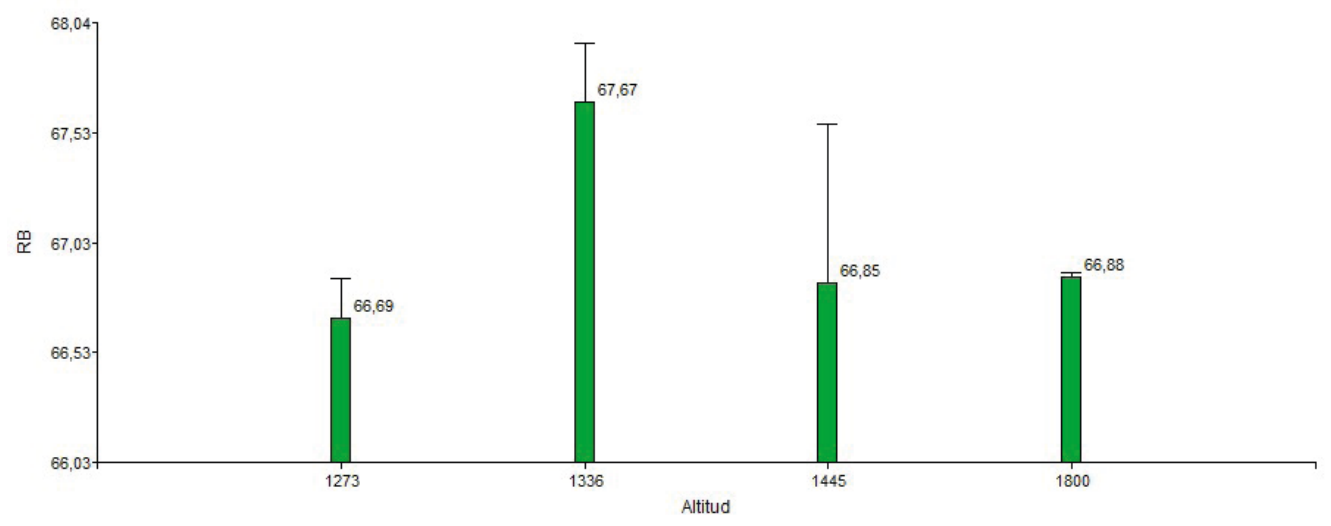

Fuente: autores 
Para determinar la asociación entre estas variables se interpretaron los datos arrojados al correr el ACP en la tabla de autovectores en la que se registran las variables $\mathrm{pH}$ y $\% \mathrm{H}$ con los valores positivos más altos, permitiendo asociar la presencia de un alto contenido de $\mathrm{MO}$ con estas dos variables. Con respecto a la $\mathrm{T}^{\circ}$ y a la CIC que registra el valor negativo más alto podemos analizar una asociación negativa con la variable MO. $\mathrm{Al}$ continuar con el análisis del porcentaje de asociación de las variables, se evidencia un $73 \%$ de asociación con respecto a los dos primeros componentes $(\% \mathrm{H} \mathrm{y} \mathrm{pH})$ frente a la variable MO, resultado que demuestra la alta asociación entre la dinámica de estas variables y la influencia en el aumento o reducción de la MO en los suelos.

Figura 4. Análisis de componentes principales del \%MO con respecto a las variables de $\mathrm{pH}, \mathrm{CIC}, \mathrm{T}^{\circ} \mathrm{y} \% \mathrm{H}$

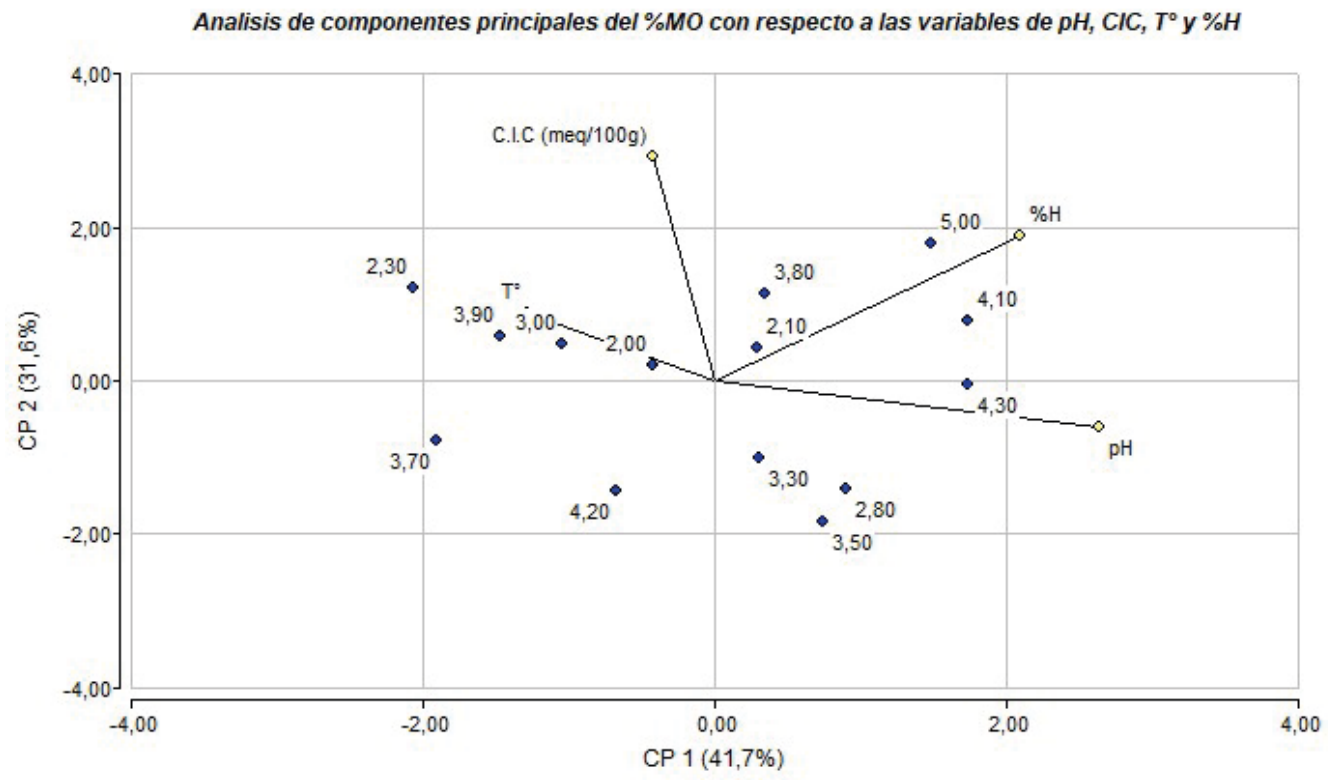

Fuente: autores 
INDAGA?EE e-ISSN: 2357-5042 • Número 8 (2020) • Universidad de Ibagué • doi: https://doi.org/10.35707/indagare/811

\section{Potencial uso}

El método de muestreo y de procesamiento de muestras implementados en la investigación permitieron conocer el contenido de la MO en las fincas muestreadas, con un promedio de contenido de 3,4\%. Los resultados de la presente investigación son la base para continuar con muestreos en el tiempo y el espacio de la MO como un importante indicador de la calidad y salud de suelos.

\section{Ficha técnica del proyecto}

Título: Evaluación de la materia orgánica con respecto a la altitud en sistemas agrícolas del Tolima.

PRIT: Conservación y aprovechamiento ecoeficiente de los recursos naturales y el medio ambiente.

Código del proyecto: $19-515-$ INT.

Palabras claves: Respiración basal, altitud, humedad, indicador.

Grupo de investigación: Naturatu.

Investigador principal: Blanca Myriam Salguero Londoño.

Correo electrónico: blanca.salguero@unibague.edu.co

\section{Referencias}

Acevedo-Osorio, A., Angarita Leiton, A., León Duran M. V., \& Franco Quiroga, K. L. (2017). Sustentabilidad y variabilidad climática: acciones agroecológicas participativas de adaptación y resiliencia socioecológica en la región alto-andina colombiana. Luna Azul, 44, 6-26. Recuperado de http://190.15.17.25/lunazul/index.php?option=com_content\&view=article\&id=217

Esperbent, C. (2018). El resguardo del suelo se transformó en el reto del siglo. Revista de Investigaciones Agropecuarias, 44(1), 10-17. Recuperado de http://ria.inta.gob.ar/contenido/el-resguardo-del-suelose-transformo-en-el-reto-del-siglo

Farrus-Miró, E. M. A. (2016). Influencia de la fertilización sobre la actividad biológica del suelo estudio comparativo de diferentes fuentes de materia orgánica (Tesis doctoral, Universitat de les Illes Balears, España). Recuperado de http://hdl.handle.net/11201/4474

García Galvis, J., \& Ballesteros González, M. I. (2005). Evaluación de parámetros de calidad para la determinación de carbono orgánico en suelos. Revista Colombiana de Química, 34(2), 201-209. Recuperado de https://revistas.unal.edu.co/index.php/rcolquim/article/view/820

Puerta Quintero, G. I., González Rizo, F. O., Correa Piedrahita, A., Álvarez Lizcano, I., E., Ardila Calderón, J. A., Girón Ospina, O. E.,...Montoya, D. F. (2016). Diagnóstico de la calidad del café según altitud, suelos y beneficio en varias regiones de Colombia. Cenicafé, 67(2), 15-51. Recuperado de https:// www.cenicafe.org/es/publications/2.Diagnostico.pdf 